\title{
Fixed Points in Grassmannians with Applications to Economic Equilibrium
}

\author{
Hans Keiding \\ Department of Economics, University of Copenhagen, Denmark \\ Email: hans.keiding@econ.ku.dk
}

\begin{abstract}
In some applications of equilibrium theory, the fixed point involves not only a state and a value of a parameter in the dual of the state space, but also a particular subspace of the state space. Since the set of all subspaces of a finite-dimensional Euclidean space has a structure which does not allow immediate application of fixed point theorems, the problem must be reformulated using a suitable parametrization of subspaces. One such parametrization, the Plücker coordinates, is used here to prove a general equilibrium existence theorem. Applications to economic problems involving hierarchies of consumers or incomplete markets with real assets are outlined.
\end{abstract}

Keywords: Existence, hierarchic equilibrium, pseudoequilibrium, Plücker coordinates

\section{Introduction}

An equilibrium problem in its simplest version is given by a map $F: K \times K \rightarrow \mathbb{R}$, where $K$ is a closed and bounded subset of some topological vector space, with the property that $F(x, x) \geq 0$ for all $x \in K$, and the task is to find an equilibrium, which is an element $x^{0} \in K$ such that $F(x, y) \geq 0$ for all $y \in K$ (Blum and Oettli [1], Bianchi and Schaible [2], Iusem [3]). In the recent literature, the discussion has been extended to more general versions of equilibrium problems, see e.g. Ansari and Flores-Bazán [4], Lin and $\mathrm{Yu}[5]$.

In some instances of equilibrium problems, notably those occurring in economic theory, the equilibrium involves not only a state $x^{0}$ but also a vector subspace chosen from a large family of such subspaces. Since the space of all subspaces of given dimension does not lend itself easily to classical fixed-point arguments, one must use roundabout methods to ascertain the existence of an equilibrium. In the approach given below, we use Plücker coordinates of vector subspaces combined with assumptions which allow us to stay away from situations where this parametrization breaks down.

The paper is organized as follows: Sect. 2 contains the main theorem and its proof, and Sect. 3 some extensions of the main theorem. In the following two sections, some applications are given, the first one dealing with economic equilibria with a hierarchy of agents, and the second one treating financial equilibria under conditions of incomplete markets with real assets. The final Sect. 7 contains a proof of a lemma used in Sect. 2.

\section{The Main Theorem}

Let $X, Y$ be subsets of $\mathbb{R}^{n}$. A multimap $\phi: X \rightrightarrows Y$ is a map from $X$ to $2^{Y}$, the set of subsets of $Y$, and the graph of $\phi$ is the set $\{(x, y) \in X \times Y \mid y \in \phi(x)\}$. Let $\mathbb{L}_{k}^{n}$ denote the set of $k$-dimensional subspaces of $\mathbb{R}^{n}$.

Definition 1 (a) A (one-person) equilibrium problem is an array $\mathcal{E}=(X, f, g, \phi, \mathbb{L})$, where

(i) $X \subset \mathbb{R}^{n}$ is nonempty, compact and convex,

(ii) $f=\left(f_{1}, \ldots, f_{k}\right): X \rightarrow X^{k}$ is continuous,

(iii) $g: X \times X \rightarrow \mathbb{R}^{n}$ is continuous, $g(X \times X) \subset X$, and $g(\cdot, x)$ is linear for each $x \in X$,

(iv) $\phi: X \rightrightarrows \mathbb{R}^{n}$ is a multimap with open graph such that $g(x, x) \notin \phi(x)$ for each $x \in X$, and

(v) $\mathbb{L} \subseteq \mathbb{L}_{k}^{n}$ is such that $f_{1}(x), \ldots, f_{k}(x) \in L$ for some $L \in \mathbb{L}$, all $x \in X$. 
(b) An equilibrium is a pair $\left(x^{0}, L^{0}\right) \in X \times \mathbb{L}_{k}^{n}$ such that $f_{j}\left(x^{0}\right) \in L^{0}, j=1 \ldots, k, g\left(x^{0}, x^{0}\right) \in L^{0}$ and $\phi\left(x^{0}\right) \cap L^{0}=\emptyset$.

Theorem 1 Let $\mathcal{E}=(X, f, g, \phi, \mathbb{L})$ be an equilibrium problem. Assume that there is $V_{0} \in \mathbb{L}_{k}^{n}$ such that for all $x \in X$, if $\phi(x)=\emptyset$, then the vectors $f_{1}(x), \ldots, f_{k}(x)$ are linearly independent, and $f_{j}(x) \notin V_{0}$, some $j$. Then there is an equilibrium in $\mathcal{E}$.

The proof of Theorem 1 relies on a suitable parametrization of $\mathbb{L}_{k}^{n}$. Let $\left(v_{1}, \ldots, v_{k}\right) \in \mathbb{R}^{n}$ be a basis of a $k$-dimensional subspace of $\mathbb{R}^{n}$. The vector of determinants of all $k \times k$ submatrices of the $k \times n$ matrix

$$
\left(\begin{array}{cccc}
v_{11} & v_{12} & \ldots & v_{1 n} \\
\vdots & \vdots & & \vdots \\
v_{k 1} & v_{k 2} & \ldots & v_{k n}
\end{array}\right)
$$

consisting of $k$ (not necessarily distinct) columns $i_{1}, \ldots, i_{k}$ is an array of $n^{k}$ numbers, not all 0 . Another basis of this subspace will give the same $n^{k}$-tuple of numbers except for multiplication by a real number, thus defining a point in $\mathbb{P}^{n^{k}-1}$, real projective space of dimension $n^{k}-1$, called the Plücker (or Grassmann) coordinates of the subspace (cf. e.g. Griffiths and Harris [6], pp. 209 - 211). The subspace is uniquely determined by its Plücker coordinates.

Define the mapping $W:\left(\mathbb{R}^{n}\right)^{k} \rightarrow \mathbb{R}^{n^{k}}$ by

$$
W_{\left(i_{1}, \ldots, i_{k}\right)}\left(v_{1}, \ldots, v_{k}\right)=\left|\begin{array}{ccc}
v_{1 i_{1}} & \ldots & v_{1 i_{k}} \\
\vdots & & \vdots \\
v_{k i_{1}} & \ldots & v_{k i_{k}}
\end{array}\right|
$$

(the determinant of the submatrix determined by columns $i_{1}, \ldots, i_{k}$ ). We shall use the following lemma, the result of which is not new, but for selfcontainedness a proof is given in Section 7 below.

Lemma 1 For each $\left(i_{1}, \ldots, i_{k}\right)$, the map $u^{\left(i_{1}, \ldots, i_{k}\right)}: \mathbb{R}^{n^{k}} \rightarrow\left(\mathbb{R}^{n}\right)^{k}$ defined by

$$
\begin{aligned}
u_{j}^{\left(i_{1}, \ldots, i_{k}\right)}(z) & =\left(u_{j 1}, \ldots, u_{j n}\right), \\
u_{j h} & = \begin{cases}z_{i_{1}, \ldots, i_{j-1}, h, i_{j+1}, \ldots, i_{k}} & h \notin\left\{i_{1}, \ldots, i_{j-1}, i_{j+1}, \ldots, i_{k}\right\}, \\
0 & \text { otherwise }\end{cases}
\end{aligned}
$$

for $j=1, \ldots, k$, is linear, and

(i) if $z_{i_{1}, \ldots, i_{k}} \neq 0$, then $u_{1}^{\left(i_{1}, \ldots, i_{k}\right)}(z), \ldots, u_{k}^{\left(i_{1}, \ldots, i_{k}\right)}(z)$ span a $k$-dimensional subspace of $\mathbb{R}^{n}$,

(ii) $W\left(u^{\left(i_{1}, \ldots, i_{k}\right)}(z)\right)=z$ if $z \in W\left(\left(\mathbb{R}^{n}\right)^{k}\right)$ and $z_{i_{1}, \ldots, i_{k}}=1$.

In the sequel, we assume that the subspace $V_{0}$ in Theorem 1 has the form

$$
V_{0}=\left\{z=\left(z_{1}, \ldots, z_{n}\right) \in \mathbb{R}^{n} \mid z_{h}=0, h>n-k\right\}
$$

This does not restrict the generality since it can be obtained by choosing a suitable basis for $\mathbb{R}^{n}$. For any $x \in X$, we let $V_{x}=\operatorname{span}\left(\left\{f_{1}(x), \ldots, f_{k}(x)\right\}\right)$ be the subspace spanned by the vectors $f_{1}(x), \ldots, f_{n}(x)$, and let $V_{x}^{\perp}$ be its orthogonal complement (in $\mathbb{R}^{m}$ ).

Lemma 2 Suppose that $\phi(x)=\emptyset$. Then $\operatorname{dim} V_{x}^{\perp}=n-k$, and

$$
W_{(1, \ldots, n-k)}\left(u_{1}, \ldots, u_{n-k}\right) \neq 0
$$

for any basis $\left(u_{1}, \ldots, u_{n-k}\right)$ of $V_{x}^{\perp}$.

Proof: For $u_{1}, \ldots, u_{n-k}$ a basis of $V_{x}^{\perp}, W_{(1, \ldots, n-k)}\left(u_{1}, \ldots, u_{n-k}\right)=0$ implies that the $(n-k)$ vectors $u_{r 1}, \ldots, u_{r, n-k}, r=1, \ldots, n-k$, are not linearly independent, so that $V_{x}^{\perp}$ contains a vector $u \neq 0$ with 
$u_{h}=0$ for $h=1, \ldots, n-k$, and consequently, every $z$ with $z_{h}=0$ for $h>n-k$ must belong to $V_{x}$, a contradiction.

Define the sets

$$
\begin{aligned}
& \mathbb{B}_{+}=\left\{w \in \mathbb{R}^{n^{n-k}} \mid\|w\| \leq 1, w_{1, \ldots, n-k} \geq 0\right\} \\
& \mathbb{S}_{+}=\left\{w \in \mathbb{B}_{+} \mid\|w\|=1\right\} .
\end{aligned}
$$

In the proof, $\mathbb{B}_{+}$is used as parameter space for subspace selection; clearly, $\mathbb{B}_{+}$is convex and compact.

Let $\Sigma$ be the set of ordered $(n-k)$-tuples $\sigma=\left(i_{1}, \ldots, i_{n-k}\right)$ from $\{1, \ldots, n\}$, and for each $w \in \mathbb{B}_{+} \backslash\{0\}$, let $\Sigma_{w}$ be the subset of $\Sigma$ consisting of all $\sigma=\left(i_{1}, \ldots, i_{n-k}\right)$ such that $w_{\sigma}=w_{i_{1}, \ldots, i_{n-k}} \neq 0$. For each $\sigma \in \Sigma_{w}$, we define the $\sigma$-normalized map $\hat{u}^{\sigma}:\left\{w \in \mathbb{B}_{+} \backslash\{0\} \mid \sigma \in \Sigma_{w}\right\} \rightarrow\left(\mathbb{R}^{n}\right)^{n-k}$ by

$$
\hat{u}^{\sigma}(w)=u^{\sigma}\left(w_{\sigma}^{-1} w\right) .
$$

Then $\left(\hat{u}_{j}^{\sigma}(w)\right)_{j=1}^{n-k}$ is a basis for an $(n-k)$-dimensional subspace of $\mathbb{R}^{n}$ associated with $w$. We write $\hat{u}^{\sigma}(w)$ as $\hat{u}^{\sigma}(w)=\left(\hat{u}_{1}^{\sigma}(w), \ldots, \hat{u}_{n-k}^{\sigma}(w)\right.$ with $\hat{u}_{j}^{\sigma}(w)=\left(u_{j 1}^{\sigma}(w), \ldots, \hat{u}_{j n}^{\sigma}(w)\right)$ for $j=1, \ldots, n-k$.

In the following, $\varepsilon>0$ is chosen arbitrarily.

Lemma 3 Let the multimaps $\gamma^{\varepsilon, \sigma}, \gamma^{\varepsilon}: X \times \mathbb{B}_{+} \rightrightarrows X$ be given by

$$
\begin{aligned}
& \gamma^{\varepsilon, \sigma}(x, w)=\left\{x^{\prime} \in X \mid-\varepsilon<\hat{u}_{j}^{\sigma}(w) \cdot g\left(x^{\prime}, x\right)<\varepsilon, j=1, \ldots, n-k\right\}, \\
& \gamma^{\varepsilon}(x, w)= \begin{cases}\operatorname{conv}\left(\left\{\gamma^{\varepsilon, \sigma}(x, w) \mid \sigma \in \Sigma_{w}\right\}\right) & w \in \mathbb{S}_{+}, \\
X & \text { otherwise, }\end{cases}
\end{aligned}
$$

Then $\gamma^{\varepsilon}$ has open graph and convex values.

Proof: Choose $(x, w) \in \mathbb{B}_{+}$and $y \in \gamma^{\varepsilon}(w)$, arbitrarily. For each $\sigma \in \Sigma$ there are open neighborhoods $U_{\sigma}$ of $(x, w)$ and $G_{\sigma}$ of $y$ such that

$$
W_{\sigma} \times G_{\sigma} \subset \operatorname{Graph} \gamma^{\varepsilon, \sigma},
$$

and if $W=\cap_{\sigma} W_{\sigma}, G=\cap_{\sigma} G_{\sigma}$, then $W$ and $G$ are open neighborhoods of $(x, w)$ and $y$ with $W \times G \subset$ Graph $\gamma^{\varepsilon}$. This shows that $\gamma^{\varepsilon}$ has open graph. Convexity of $\gamma^{\varepsilon}(x, w)$ follows from the definition of $\gamma^{\varepsilon}$ and the linearity of $g(\cdot, x)$.

Lemma 4 For $i=1, \ldots, m$, the multimap $\psi^{\varepsilon}: X \times \mathbb{B}_{+} \rightrightarrows X$ given by

$$
\psi^{\varepsilon}(x, w)= \begin{cases}\gamma^{\varepsilon}(x, w) & g(x, x) \notin c l \gamma^{\varepsilon}(w) \\ \gamma^{\varepsilon}(x, w) \cap \phi(x) & \text { otherwise. }\end{cases}
$$

has open graph and convex values, and $x \notin \psi^{\varepsilon}(x, w)$ for each $(x, w) \in X \times \mathbb{B}_{+}$.

Proof: Let $(x, w) \in X \in \mathbb{B}_{+}$and $y \in \psi^{\varepsilon}(x, w)$ be arbitrary. Choose an open neighbourhood $G$ of $y$; if $g(x, x) \notin \operatorname{cl} \gamma^{\varepsilon}(w)$, then there is a neighborhood $U$ of $(x, w)$ such that $\psi^{\varepsilon}\left(x^{\prime}, w^{\prime}\right)=\gamma^{\varepsilon}\left(w^{\prime}\right)$ for all $\left(x^{\prime}, w^{\prime}\right) \in U$, so that $\psi^{\varepsilon}$ has open graph on all such $(x, w)$ by Lemma 3 . If $g(x, x) \in \operatorname{cl} \gamma^{\varepsilon}(w)$, then $y \in \phi(x)$, and there is an open neighborhood of $(x, w)$ such that $\phi\left(x^{\prime}\right)$ intersects $G$. It follows that $\phi^{\varepsilon}$ is lower hemicontinuous on all of $X \times \mathbb{B}_{+}$.

Convexity of $\psi^{\varepsilon}(x, w)$ for each $(x, w) \in X \times \mathbb{B}_{+}$is obvious. Also for each $(x, w) \in X \times \mathbb{B}_{+}$, we have that $\psi^{\varepsilon}(x, w) \subset \gamma^{\varepsilon}(x, w)$, and if $x \in \operatorname{cl} \gamma^{\varepsilon}(x, w)$, then $\psi^{\varepsilon}(x, w) \subset \phi(x)$. Since $g(x, x) \notin \phi(x)$ for all $x \in X$, the statement in the lemma follows.

Let $W(\mathbb{L}) \subseteq \mathbb{S}_{+}$be the set of points $W\left(v_{1}, \ldots, v_{n-k}\right)$ where $\left(v_{1}, \ldots, v_{n-k}\right)$ is a basis of a subspace $L^{\perp}$ with $L \in \mathbb{L}$.

Lemma 5 The multimap $\Omega: X \rightrightarrows \mathbb{S}_{+}$defined by

$$
\Omega(x)=\left\{w \in W(\mathbb{L}) \mid \exists v_{1}, \ldots, v_{d-k} \in f(x)^{\perp}, W\left(v_{1}, \ldots, v_{n-k}\right)=w\right\}
$$

has closed graph and nonempty values. 
Proof: Nonemptiness of $\Omega(x)$ for each $x$ is obvious, as $f(x)$ consists of at most $k$ linearly independent vectors. Let $\left(x^{\nu}, w^{\nu}\right)_{\nu=1}^{\infty}$ be a sequence in the graph of $\Omega$ converging to some $\left(x^{0}, w^{0}\right) \in X \times \mathbb{S}_{+}$. For each $\nu$, choose an orthonormal basis $\left(v_{1}^{\nu}, \ldots, v_{n-k}^{\nu}\right)$ for an $(n-k)$-dimensional subspace of $\mathbb{R}^{n}$ with

$$
v_{r}^{\nu} \cdot f_{j}\left(x^{\nu}\right)=0, r=1, \ldots, n-k, j=1, \ldots, n,
$$

and $\left(W\left(v_{1}^{\nu}, \ldots, v_{n-k}^{\nu}\right)=w^{\nu}\right.$. Taking subsequences if necessary, it may be assumed that the sequence $\left(v^{\nu}\right)_{\nu=1}^{\infty}$ with $v^{\nu}=\left(v_{1}^{\nu}, \ldots, v_{n-k}^{\nu}\right)$ converges to some $v^{0}=\left(v_{1}^{0}, \ldots, v_{n-k}^{0}\right) \in\left(\mathbb{R}^{n}\right)^{n-k}$. Since $W\left(v^{0}\right)=w^{0}$ by continuity of $W$, the family $\left\{v_{1}^{0}, \ldots, v_{n-k}^{0}\right\}$ spans an $(n-k)$-dimensional subspace of $\mathbb{R}^{n}$. Continuity of $f$ implies that

$$
v_{r}^{0} \cdot f_{j}\left(x^{0}\right)=0, r=1, \ldots, n-k, j=1, \ldots, k,
$$

so that $w^{0} \in \Omega\left(p^{0}\right)$.

Lemma 6 There is a multimap $H \times \operatorname{conv} \Omega: X \times \mathbb{B}_{+} \rightrightarrows X$ with closed graph and nonempty convex values such that $H(x, w) \subset \psi^{\varepsilon}(x, w)$ whenever $\psi^{\varepsilon}(x, w) \neq \emptyset$.

Proof: The set $\operatorname{Dom} \psi^{\varepsilon}=\{(x, w) \mid \psi(x, w) \neq \emptyset\}$ is open, and by Michael's selection theorem (Michael $[7])$, there is a continuous map $h: \operatorname{Dom} \psi^{\varepsilon} \rightarrow X$ such that $h(x, w) \in \psi^{\varepsilon}(x, w)$ for $(x, w) \in \operatorname{Dom} \psi^{\varepsilon}$. Let $H: X \times \mathbb{B}_{+} \rightrightarrows X$ be given by

$$
H(x, w)= \begin{cases}h(x, w) & (x, w) \in \operatorname{Dom} \psi^{\varepsilon} \\ X & \text { otherwise. }\end{cases}
$$

Then $H$ has the desired properties.

Now we may apply a fixed point theorem to obtain an approximate equilbrium. The lemma uses the approach of Gale and Mas-Colell [8].

Lemma 7 There is $\left(x^{\varepsilon}, w^{\varepsilon}\right) \in X \times \mathbb{B}_{+}$, such that

(i) $\psi^{\varepsilon}\left(x^{\varepsilon}, w^{\varepsilon}\right)=\emptyset$,

(ii) $w^{\varepsilon} \in \Omega\left(x^{\varepsilon}, w^{\varepsilon}\right)$ and $w^{\varepsilon} \in W(\mathbb{L})$.

Proof: The multimap

$$
H \times \operatorname{conv} \Omega: X \times \mathbb{B}_{+} \rightrightarrows X \times \mathbb{B}_{+},
$$

given by Lemma 6 where conv $\Omega$ takes $(x, w)$ to the convex hull of $\Omega(w)$, is upper hemicontinuous with nonempty closed and convex values, and by the fixed-point theorem of Kakutani [9], there is $\left(x^{\varepsilon}, w^{\varepsilon}\right)$ such that

$$
x^{\varepsilon} \in H\left(x^{\varepsilon}, w^{\varepsilon}\right), w^{\varepsilon} \in \operatorname{conv} \Omega\left(x^{\varepsilon}\right) .
$$

From the irreflexivity of the multimaps $\psi^{\varepsilon}\left(x^{\varepsilon}, w^{\varepsilon}\right)=\emptyset$, so that that property (i) of the lemma is satisfied. To show that property (ii) holds, assume to the contrary that $w^{\varepsilon}$ does not belong to $\Omega\left(x^{\varepsilon}\right)$, i.e. that $w^{\varepsilon}$ is a proper convex combination of points in $\Omega\left(x^{\varepsilon}\right)$. Then $w^{\varepsilon}$ belongs to $\mathbb{B}_{+} \backslash \mathbb{S}_{+}$, and by definition, $\gamma^{\varepsilon}\left(x^{\varepsilon}, w^{\varepsilon}\right)=X$. It follows that $\phi\left(x^{\varepsilon}\right)=\emptyset$, so that $x^{\varepsilon} \in E$. However, by Lemma $2, \Omega\left(x^{\varepsilon}\right)$ then consists of a unique point in $w \in \mathbb{S}_{+}$with $w_{(1, \ldots, l-k)}>0$, and it follows that $w \in W(\mathbb{L})$.

Now we are in a position to prove the main theorem.

Proof of Theorem 1: Let $\left(\varepsilon_{\nu}\right)_{\nu=1}^{\infty}$ be a decreasing sequence of numbers converging to 0 . For each $\nu$, choose a pair $\left(x^{\varepsilon_{\nu}}, w^{\varepsilon_{\nu}}\right)$ satisfying (i) and (ii) of Lemma 7 . Since $w^{\varepsilon_{\nu}} \in \Omega\left(x^{\varepsilon}\right) \cap \mathbb{S}_{+}$, it defines a $(n-k)$-dimensional subspace of $\mathbb{R}^{n}$; let $L^{\varepsilon_{\nu}}$ be its orthogonal complement. Then $f\left(x^{\varepsilon_{\nu}}\right) \in L^{\varepsilon_{\nu}}$ and $L^{\varepsilon_{\nu}} \in \mathbb{L}$.

Identify the elements of $\mathbb{L}_{k}^{n}$ with their intersections with the compact set $X$, so that the PainlevéKuratowski and Hausdorff topologies on $\mathbb{L}_{k}^{n}$ are identical. Using the definition of $\psi_{\nu}^{\varepsilon}$, we get that

$$
\gamma^{\varepsilon_{\nu}}\left(x^{\varepsilon_{\nu}}, w^{\varepsilon_{\nu}}\right) \cap \phi(x)=\emptyset
$$

Therefore, $d\left(x^{\varepsilon_{\nu}}, L^{\varepsilon_{\nu}}\right) \leq \varepsilon_{\nu}$ (where $d$ is distance from the point $x^{\varepsilon_{\nu}}$ to the set $\left.X \cap L^{\varepsilon_{\nu}}\right)$. 
Since $X \times \mathbb{L}$ is compact, we may assume that the sequence $\left(x^{\varepsilon_{\nu}}, L^{\varepsilon_{\nu}}\right)_{\nu=1}^{\infty}$ converges to some $\left(x^{0}, L^{0}\right) \in$ $X \times \mathbb{L}$. Clearly, $g\left(x^{0}, x^{0}\right) \in L^{0}$ and $f\left(x^{0}\right) \in\left(L^{0}\right)^{k}$.

We check that $\left(x^{0}, L^{0}\right)$ has the properties stated in Theorem 1 . Suppose that $x \in \phi\left(x^{0}\right)$ belongs to $L^{0}$. Since $\phi$ has open graph, there is are open neighborhoods $N_{x}$ of $x$ and $N_{x^{0}}$ of $x^{0}$ such that $N_{x} \subset \phi\left(x^{\prime}\right)$ for $x^{\prime} \in N_{x^{0}}$. But $L^{\varepsilon_{\nu}} \cap N_{x} \neq \emptyset$ for large enough $n$, and $x^{\varepsilon_{\nu}} \rightarrow x^{0}$, and we get a contradiction.

\section{Extensions of the Main Theorem}

The result of Theorem 1 can be somewhat improved, since the assumption of linear independence of the vectors $f_{1}(x), \ldots, f_{k}(x)$ can be replaced by a weaker one, at least if we allow $\mathbb{L}$ to be all of $\mathbb{L}_{k}^{n}$. We then get the folkowing version of the main theorem.

Theorem 2 Let $\mathcal{E}=(X, f, g, \phi, \mathbb{L})$ be an equilibrium problem. Assume that the set $E=\{x \in X \mid \phi(x)=$ $\emptyset\}$ is finite, and there is $V_{0} \in \mathbb{L}_{k}^{n}$ such that for each $x \in E, f_{j}(x) \notin V_{0}$ for some $j$. Then there is an equilibrium in $\mathcal{E}$.

Proof: Let $E=\left\{x_{1}, \ldots, x_{p}\right\}$, choose $\varepsilon>0$ so small that there are mutually disjoint $\varepsilon$-neighborhoods $U_{t}^{\varepsilon}$ of $x_{t}, t=1, \ldots, p$. For each $t$ choose $k$ linearly independent vectors $v_{1}^{\varepsilon}, \ldots, v_{k}^{\varepsilon}$ such that

$$
\left\|v_{j}^{\varepsilon}-f_{j}\left(x_{t}\right)\right\|<\frac{\varepsilon}{2 n}, j=1, \ldots, k .
$$

The family $\left\{U_{0}^{\varepsilon}, U_{1}^{\varepsilon}, \ldots, U_{p}^{\varepsilon}\right\}$, where $U_{0}^{\varepsilon}$ is open and $X \backslash \cup_{t=1}^{p} U_{t}^{\varepsilon} \subset U_{0}^{\varepsilon} \subset X \backslash E$, is an open covering of $X$. Let $\left(\psi_{t}^{\varepsilon}\right)_{t=0}^{p}$ be a partition of unity subordinated to this covering, and define the map $f^{\varepsilon}: X \rightarrow\left(\mathbb{R}^{n}\right)^{k}$ by

$$
f_{j}^{\varepsilon}(x)=\sum_{t=1}^{p} \psi_{t}^{\varepsilon}(x) v_{t}^{\varepsilon}(x)+\psi_{0}^{\varepsilon} f_{j}(x), j=1 \ldots, k,
$$

for $x \in X$. Applying Theorem 1 to the problem given by $X, \mathbb{L}_{k}^{n}, \phi$ and $f^{\varepsilon}$, we find a point $x^{\varepsilon} \in X$ and a subspace $L^{\varepsilon} \in \mathbb{L}_{k}^{n}$ such that $f_{\varepsilon}(x) \in L^{\varepsilon}$ and $\psi(x) \cap L^{\varepsilon}=\emptyset$. If $x^{\varepsilon} \in X \backslash \cup_{t=1}^{p} U_{t}^{\varepsilon}$, then $x^{\varepsilon}$ has all the desired properties, and we are done. So we may assume that $x^{\varepsilon} \in U_{t}^{\varepsilon}$ for some $t \in\{1, \ldots, p\}$.

Choosing a sequence of numbers $\varepsilon>0$ going to 0 , and passing to subsequences if necessary, we obtain sequences of points $x^{\varepsilon}$ converging to some $x^{0} \in E$, and $k$-dimensional subspaces $L^{\varepsilon}$ converging to some $L^{0} \in \mathbb{L}_{k}^{n}$. By our construction $x^{0}$ and $L^{0}$ have the desired properties.

It is seen that the method of proof applied relies on the possibility of approaching the vectors $f_{j}(x), j=1, \ldots, k$ arbitrarily close by linearly independent vectors $v_{1}, \ldots, v_{k}$, for each $x \in E$. The assumption of finiteness of $E$ might be done away with altogether if the approximations could be chosen as continuous functions of $x$ on all of $E$.

The result of Theorem 1 (or Theorem 2) can be extended easily so as to cover the case of several multimaps $\phi_{1}, \ldots, \phi_{r}$ instead of a single one.

Definition 2 A many-person equilibrium problem is an array $\left(X, f,\left(g_{h}, \phi_{h}\right)_{h=1}^{r}, \mathbb{L}\right)$, where

(i) $X \subset \mathbb{R}^{n}$ is nonempty, compact and convex,

(ii) $f=\left(f_{1}, \ldots, f_{k}\right): X \rightarrow X^{k}$ is continuous,

(iii) for $h=1, \ldots, r, g_{h}: X \times X^{r} \rightarrow \mathbb{R}^{n}$ is continuous, $g_{h}\left(X \times X^{r}\right) \subset X$, and $g_{h}(\cdot, x)$ is linear for each $x \in X^{r}$,

(iv) for $h=1, \ldots, r, \phi_{h}: X \rightrightarrows \mathbb{R}^{n}$ is a multimap with open graph such that $g_{h}(x, x) \notin \phi_{h}(x)$ for each $x \in X$, and

(v) $\mathbb{L} \subseteq \mathbb{L}_{k}^{n}$ is such that $f_{1}(x), \ldots, f_{k}(x) \in L$ for some $L \in \mathbb{L}$, all $x \in X$.

An equilibrium is a pair $\left(x^{0}, L^{0}\right) \in X^{r} \times \mathbb{L}_{k}^{n}$ such that $x^{0}=\left(x_{1}^{0}, \ldots, x_{r}^{0}\right) \in L^{0}, f_{j}\left(x^{0}\right) \in L^{0}, j=1, \ldots, k$, $g_{h}\left(x_{h}^{0}, x^{0}\right) \in L^{0}$ and $\phi_{h}\left(x_{h}^{0}\right) \cap L^{0}=\emptyset$ for $h=1, \ldots, r$.

Theorem 3 Let $\mathcal{E}=\left(X, f,\left(g_{h}, \phi_{h}\right)_{h=1}^{r}, \mathbb{L}\right)$ be a many-person equilibrium problem. Assume that the set $E=\left\{x \in X \mid \phi_{h}(x)=\emptyset, h=1, \ldots, r\right\}$ is finite, and there is $V^{0} \in \mathbb{L}_{k}^{n}$ such that for each $x \in E$, $f_{j}(x) \notin V^{0}$, some $j=1, \ldots, k$. Then there is an equilibrium in $\mathcal{E}$. 
The proof of Theorem 1 works also in this case, and the only extension needed is a reformulation of Lemma 6 so as to obtain a multimap $H: X^{r} \times \mathbb{B}_{+} \rightrightarrows X^{r}$ with the property that $H_{i}\left(x_{1}, \ldots, x_{r}, w\right) \subset$ $\psi_{i}^{\varepsilon}\left(x_{i}, w\right)$ whenever $\psi_{i}^{\varepsilon}\left(x_{i}, w\right) \neq \emptyset$, where the multimaps $\psi_{1}^{\varepsilon}, \ldots, \psi_{r}^{\varepsilon}$ are constructed from $\phi_{1}, \ldots, \phi_{r}$ as in Lemmas 3 and 4 above.

\section{Applications (1): Hierachic Equilibria}

We consider an exchange economy with $m$ consumers and $l$ commodities, cf. Debreu [10]. Each consumer $i$ is characterized by the pair $\left(Z_{i}, P_{i}\right)$. Here $Z_{i} \subset \mathbb{R}^{n}$ is a set of feasible net trades in the $n$ commodities, and $P_{i}: Z_{i} \rightrightarrows Z_{i}$ is a multimap describing the preferences of consumer $i$ in the sense that $z_{i}^{\prime} \in P_{i}\left(z_{i}\right)$ means that $z_{i}^{\prime}$ is considered as better than $z_{i}$ by consumer $i$. We assume (here and in the next section) tha the economy is well-behaved in the sense that for each $i$,

(a) $Z_{i}$ is closed, convex and bounded from below, $0 \in \operatorname{int} Z_{i}$ and $Z_{i}+\mathbb{R}_{+}^{l} \subseteq Z_{i}$,

(b) $z_{i} \notin P_{i}\left(z_{i}\right)$ for all $z_{i} \in Z_{i}$ (irreflexivity),

(c) $P_{i}\left(z_{i}\right)$ is a convex set, all $z_{i} \in Z_{i}$ (convexity),

(d) the graph of $P_{i}$ is open in $Z_{i} \times Z_{i}$ (continuity).

A price system in the economy is a vector $p \in \Delta=\left\{p \in \mathbb{R}_{+}^{n} \mid \sum_{h=1}^{n} p_{h}=1\right\}$. A competitive equilibrium is a pair $\left(z_{1}, \ldots, z_{m}, p\right) \in \mathbb{R}^{l m} \times \Delta$ such that $\sum_{i=1}^{m} z_{i}=0$ (aggregate balance of net trades) and $p \cdot z_{i} \leq 0, P_{i}\left(z_{i}\right) \cap\left\{z_{i}^{\prime} \in Z_{i} \mid p \cdot z_{i}^{\prime} \leq 0\right\}=\emptyset, i=1, \ldots, m$ (individual optimality).

Definition 3 Let $\mathcal{L}=\left(L^{1}, \ldots, L^{k}\right)$ with $L^{k} \subset L^{k-1} \subset \cdots \subset L^{1}=\mathbb{R}^{l}$ be a chain of subspaces of $\mathbb{R}^{l}$ where for each $j$ there is $i$ such that 0 belongs to the relative interior of $Z_{i} \cap L^{j}$. A hierarchic equilibrium relative to $\mathcal{L}$ is an array $\left(z_{1}, \ldots, z_{m},\left(p^{j}\right)_{j=1}^{k}\right)$, where $z_{i} \in Z_{i}, i=1, \ldots, m$, and $p^{j} \in \Delta, j=1, \ldots, n$, satisfying the following conditions:

(i) $\sum_{i=1}^{m} z_{i}=0$,

(ii) For $i=1, \ldots, m$, if $\min _{z_{i}^{\prime} \in Z_{i}} p^{j} \cdot z_{i}^{\prime}<0$, then $z_{i} \in L^{j}, p^{j} \cdot z_{i}=0$ and $P_{i}\left(z_{i}\right) \cap\left\{z_{i}^{\prime} \in Z_{i} \cap L^{j} \mid\right.$ $\left.p^{j} \cdot z_{i} \leq 0\right\}=\emptyset$.

Intuitively, in a hierarchical equilibrium consumers and commodities are split into classes, so that members of classes with higher index are too poor to buy all the commodities available to those in classes with a lower index. Hierarchic equilibria were introduced by Danilov and Sotskov [11], see also Florig [12], Konovalov and Marakulin [13].

For simplicity, we consider here only hierarchies of the form $\mathcal{L}=\left(L^{1}, L^{2}\right)$ with $L^{1}=\mathbb{R}^{l}$ and $L^{2}$ a proper subspace of $\mathbb{R}^{l}$.

Theorem 4 Let $\mathcal{E}=\left(Z_{i}, P_{i}\right)_{i=1}^{m}$ be a well-behaved economy. Assume that the associated structure of subspaces is $\left(L^{1}, L^{2}\right)$. Then there exists a hierarchic equilibrium in $\mathcal{E}$.

Proof: In order to use the results of the previous section, we must define the state space $X$ and the family $\mathbb{L}$ of subspaces. For state space, we use the set of all arrays $\left(z_{1}, \ldots, z_{m}, p^{1}, p^{2}\right)$ of net trades and prices such that each $z_{i}$ belongs to a suitably bounded subset $\hat{Z}_{i}$ of $Z_{i}$, making sure that if $\sum_{i=1}^{m} z_{i} \leq 0$, then $z_{i} \in \hat{Z}_{i}$, all $i$. Next, for $j=1,2$, let $I^{j}=\left\{i \in\{1, \ldots, m\} \mid 0 \in \operatorname{ri} Z^{i} \cap L^{j}\right\}$ and $j(i)=\left\{j \mid i \in I^{j}\right\}$, and define $\mathbb{L}$ as the family $\{L\}$ consisting of a single subspace

$$
L=\underbrace{L^{1} \oplus \cdots \oplus L^{1}}_{\left|I^{1}\right| \text { copies }} \oplus \underbrace{L^{2} \oplus \cdots \oplus L^{2}}_{\left|I^{2}\right| \text { copies }} \oplus \mathbb{R}^{2 l} .
$$

The maps $f_{1}, \ldots, f_{k}$ are inessential for this application and are defined as $f_{j}(x)=0$ for $j=1, \ldots, k$, and the map $g$ is projection on the second component, $g\left(x, x^{\prime}\right)=x^{\prime}$ for all $\left(x, x^{\prime}\right) \in X^{2}$.

We now define the multimap $\phi: X \rightrightarrows X$ in several steps. For $i=1, \ldots, m$, define multimaps $\beta_{i}: \Delta^{j(i)} \rightrightarrows Z_{i}$ and $\phi_{i}: \hat{Z}_{i} \times \Delta^{j(i)} \rightrightarrows Z_{i}$ by

$$
\begin{aligned}
\beta_{i}\left(p^{j(i)}\right) & =\left\{z_{i}^{\prime} \in \hat{Z}_{i} \cap L^{j(i)} \mid p^{j(i)} \cdot z_{i}^{\prime}<0\right\}, \\
\phi_{i}\left(z_{i}, p^{j(i)}\right) & = \begin{cases}\beta_{i}\left(p^{j(i)}\right) & z_{i} \notin \operatorname{cl} \beta_{i}\left(p^{j(i)}\right), \\
\beta_{i}\left(p^{j(i)}\right) \cap P_{i}\left(z_{i}\right) & z_{i} \in \operatorname{cl} \beta_{i}\left(p^{j(i)}\right) .\end{cases}
\end{aligned}
$$


Next, define the multimaps $\phi^{j}: \hat{Z}_{1} \times \cdots \times \hat{Z}_{m} \rightrightarrows \Delta^{j}$ for $j=1,2$ by

$$
\phi^{j}\left(z_{1}, \ldots, z_{m}\right)=\left\{p^{j} \in \Delta^{j} \mid p^{j} \cdot \sum_{i \in I^{j}} z_{i}>0\right\} .
$$

For $i=1, \ldots, m$, by our construction and using the properties of $P_{i}$, we get that $\phi_{i}$ has open graph and satisfies $z_{i} \notin \phi_{i}\left(z_{i}, p^{1}\right)$, all $\left(z_{i}, p^{1}\right)$, and $\phi^{j}$ has open graph for $j=1,2$. Moreover, if $z_{i} \in \operatorname{cl} \beta_{i}\left(p^{1}\right), i \in I^{1}$, and $z_{i} \in \operatorname{cl} \beta_{i}\left(p^{2}\right), i \in I^{2}$, then $p^{1} \notin \phi^{1}\left(z_{1}, \ldots, z_{m}\right), p^{2} \notin \phi^{2}\left(z_{1}, \ldots, z_{m}\right)$. Define now $\hat{\phi}_{i}, i=1, \ldots, m$, and $\hat{\phi}^{j}, j=1,2$ by

$$
\begin{aligned}
\hat{\phi}_{i}\left(z_{i}, p^{j(i)}\right) & = \begin{cases}\phi_{i}\left(z_{i}, p^{j(i)}\right) & z_{i} \notin \phi_{i}\left(z_{i}, p^{j(i)}\right) \\
\hat{Z}_{i} & \text { otherwise }\end{cases} \\
\hat{\phi}^{j}\left(z_{1}, \ldots, z_{m}\right) & = \begin{cases}\phi^{j}\left(z_{1}, \ldots, z_{m}\right) & p^{j} \notin \phi^{j}\left(z_{1}, \ldots, z_{m}\right) \\
\hat{\Delta}^{j} & \text { otherwise }\end{cases}
\end{aligned}
$$

Then the multimap $\phi: X \rightrightarrows X$ given by

$$
\phi\left(z_{1}, \ldots, z_{m}, p^{1}, p^{2}\right)=\prod_{i \in I^{1}} \hat{\phi}_{i}\left(z_{i}, p^{1}\right) \times \prod_{i \in I^{2}} \hat{\phi}_{i}\left(z_{i}, p^{2}\right) \times \prod_{j=1}^{2} \hat{\phi}^{j}\left(z_{1}, \ldots, z_{m}\right)
$$

satisfies the properties stated in Theorem 1.

In order to apply the theorem, we consider an economy $\mathcal{E}=\left(\left(Z_{1}, P_{1}\right), \ldots,\left(Z_{m}, P_{m}\right)\right)$ for which no competitive equilibrium exists. In this case the assumptions on the mappings $f_{1}, \ldots, f_{k}$ are fulfilled trivially, and Theorem 1 gives the existence of a state $x^{0}=\left(z_{1}^{0}, \ldots, z_{m}^{0}, p_{0}^{1}, p_{0}^{2}\right)$ such that $z_{i} \in L^{2}$ for each $i \in I^{2}$ and such that $\phi_{i}\left(x^{0}\right) \cap L=\emptyset$. By the definition of $\phi$, we get that $\phi_{i}\left(z_{i}^{0}, p^{j(i)}\right)=\emptyset$ for $i=1, \ldots, m$, meaning that $p^{j(i)} \cdot z_{i} \leq 0$ and $P_{i}\left(z_{i}\right) \cap\left\{z_{i}^{\prime} \in Z_{i} \cap L^{j(i)} \mid p^{j(i)} \cdot z_{i}^{\prime} \leq 0\right\}=\emptyset$. Moreover, $\phi^{j}\left(z_{1}^{0}, \ldots, z_{m}^{0}\right)=\emptyset$ for $j=1,2$, so that $\sum_{i=1}^{m} z_{i}^{0} \leq 0$. Summing up, we have shown that $\left(z_{1}^{0}, \ldots, z_{m}^{0}, p_{0}^{1}, p_{0}^{2}\right)$ is a hierarchic equilibrium.

\section{Application (2): Pseudoequilibria in Economies with Incomplete Markets and Real Assets}

In this section we apply Theorem 2 to show existence of equilbrium in an economy with real assets and incomplete markets. For a detailed account of the theory we refer to Duffie and Shafer [14], Geanakoplos [15], Magill and Shafer [16]. More recent contributions are e.g. Zhou [17], Momi [18], and Hoelle, Pireddu and Villanacci [19].

As in the previous section, there are $m$ consumers and $l$ commodities. The latter are interpreted as contingent commodities: There are two periods of time, $t=0$ and $t=1$, and $S$ uncertain states of the world at $t=1$, and assuming a fixed number $n$ of basic goods at each date and state of the world, we get that $l=(S+1) n$.

An asset structure is given by $k$ assets $a_{1}, \ldots, a_{k} \in \mathbb{R}^{S n}$, which define the transfers of value between states that are possible in the model. For $p=\left(p_{0}, p_{1}, \ldots, p_{S}\right) \in \Delta$ a price and $z=\left(z_{0}, z_{1}, \ldots, z_{S}\right) \in \mathbb{R}^{l}$ a vector of net trades in each state, the box product

$$
p \square z=\left(p_{1} \cdot z_{1}, \ldots, p_{S} \cdot z_{S}\right)
$$

of $p$ and $z$ (where $\cdot$ denotes the inner product in $\mathbb{R}^{n}$ ) gives the values of the net trade $z$ in each of the future states.

Definition 4 An array $(z, p, L)$ with $z=\left(z_{1}, \ldots, z_{m}\right) \in \prod_{i=1}^{m} Z_{i}, p \in \Delta$, and $L \in \mathbb{L}_{k}^{n}$, is a pseudoequilibrium if

(i) $\sum_{i=1}^{m} z_{i}=0$,

(ii) for all $i, p \cdot z_{i}=0, p \square z_{i} \in L$, and $P_{i}\left(z_{i}\right) \cap\left\{z_{i}^{\prime} \in Z_{i} \mid p \cdot z_{i}^{\prime}=0, p \square z_{i}^{\prime} \in L,\right\}=\emptyset$,

(iii) $p \square a_{j} \in L, j=1, \ldots, k$. 
To explain the terminology, we notice the conditions for a pseudoequilibrium demands only that all the vectors $p \square a_{j}$ are in $L$, not that $L$ is actually spanned by these vectors. If we insisted on the spanning condition, the existence theorem below would no longer hold true, as shown by counterexamples, cf. e.g. Hart [20].

Using Theorem 1 we can prove the following result.

Theorem 5 Let the economy with real assets be well-behaved (conditions (a)-(d) of the previous section) and satisfy the strong monotonicity condition

(e) $z_{i}+\left[\mathbb{R}_{+}^{l} \backslash\{0\}\right] \subseteq P_{i}\left(z_{i}\right)$ for all $z_{i}$.

Suppose, in addition, that the set $E$ is a finite set, and for each $\left(z_{1}, \ldots, z_{m}, p\right) \in E$, the set $\left\{y \in \mathbb{R}^{S} \mid\right.$ $\left.y_{h}=0, h>k\right\}$ is not contained in the subspace spanned by $p \square a_{j}, j=1, \ldots, k$.

Then the economy has a pseudoequilibrium.

Proof: The proof consists in applying Theorem 2 to a suitably constructed problem. As the first step, we proceed as in the proof of Theorem 4 and replace each $Z_{i}$ by the compact and convex set $\hat{Z}_{i}=\left\{z_{i} \in Z_{i} \mid\right.$ $\left.z_{i} \leq \omega\right\}$, where $\omega \in \mathbb{R}_{+}^{n}$ is such that $z=\sum_{i=1}^{m} z_{i}$ with $z_{i} \in Z_{i}$ implies that $z_{h}>-\omega_{h}$ for $h=1, \ldots, l$

For each $i$, let $A_{i}$ be a compact and convex set containing all vectors $p \square z_{i}$ for $z_{i} \in Z_{i}, p \in \Delta$. Let $X=\left[\prod_{i=1}^{m} \hat{Z}_{i} \times A_{i}\right] \times \Delta \subset\left(\mathbb{R}^{n} \times \mathbb{R}^{S}\right)^{m} \times \mathbb{R}^{n}$. Clearly, $X$ is convex and compact.

To define $\mathbb{L}$ we notice that each $k$-dimensional subspace $\tilde{V}$ of $\mathbb{R}^{S}$ defines a subspace $V=\left(\mathbb{R}^{n} \times \tilde{V}\right)^{m} \times \mathbb{R}^{n}$ of $\left(\mathbb{R}^{n} \times \mathbb{R}^{S}\right)^{m} \times \mathbb{R}^{n}$. Let $\mathbb{L}$ be the set of all such subspaces $V$. Define $f_{j}: X \rightarrow X, j=1, \ldots, m$ by

$$
f_{j}(x)=\left(\left(0, p \square a_{j}\right), \ldots,\left(0, p \square a_{j}\right), 0\right), j=1, \ldots, k,
$$

and let $g: X \times X \rightarrow X$ be given by

$$
\begin{aligned}
g\left(\left(\left(z_{1}, s_{1}\right), \ldots,\left(z_{m}, s_{m}\right), p\right)\right. & ,\left(\left(z_{1}^{\prime}, s_{1}^{\prime}\right), \ldots,\left(z_{m}^{\prime}, s_{m}^{\prime}\right), p^{\prime}\right) \\
& =\left(\left(z_{1}^{\prime}, p \square z_{1}^{\prime}\right), \ldots,\left(z_{m}^{\prime}, p \square z_{m}^{\prime}\right), p^{\prime}\right) .
\end{aligned}
$$

Clearly, $f_{j}$ is continuous for each $j, g$ is continous, and $g(x,$.$) is the restriction to X$ of a linear map.

For $i=1, \ldots, m$, let $\beta_{i}, \phi_{i}: X \rightrightarrows \hat{Z}_{i}$ be defined as in (3) and (4), and define $\phi_{0}: X \rightrightarrows \Delta$ by

$$
\phi_{0}(x)=\left\{p^{\prime} \in \Delta \mid p^{\prime} \cdot \sum_{i=1}^{m} z_{i}>0\right\} .
$$

It is easily checked that $\phi_{i}$ has open graph and convex values, and that $g(x . x) \notin \phi_{i}(x)$ for all $x \in X$, $i=0,1, \ldots, m$.

We check that the assumptions of Theorem 3 are satisfied. First of all, if $x=\left(\left(z_{1}, s_{1}\right), \ldots,\left(z_{m}, s_{m}\right), p\right)$ and $\phi_{i}(x)=\emptyset$ for all $i$, then $p \cdot z_{i} \leq 0$ for $i=1, \ldots, n$, so that $\phi_{0}(x)=\emptyset$ implies that $\sum_{i=1}^{m} z_{i} \leq 0$. From and $p \cdot z_{1} \leq 0$ we get from monotonicity of preferences that $p_{h}>0$ for $h=1, \ldots, n$, so $p \cdot \sum_{i=1}^{m} z_{i}=0$ and $\left(z_{1}, \ldots, z_{m}\right)$ is a competitive equilibrium. We conclude that $\left\{x \in X \mid \phi_{i}(x)=\emptyset, i=0,1, \ldots, m\right\}$ is a finite set.

We now apply Theorem 3 . Let $\left(x^{0}, L^{0}\right) \in X \times \mathbb{L}$ be such that

$$
\begin{gathered}
f_{j}\left(x^{0}\right) \in L^{0}, j=1, \ldots, k, \\
g\left(x^{0}, x^{0}\right) \in L^{0}, \\
\phi_{i}\left(x^{0}\right) \cap L^{0}=\emptyset, i=0,1, \ldots, m .
\end{gathered}
$$

Write $x^{0}=\left(z_{1}^{0}, \ldots, z_{m}^{0}, p_{1}^{0} \square z_{1}^{0}, \ldots, p_{m}^{0} \square z_{m}^{0}, p^{0}\right)$ and

$$
L^{0}=\left\{\left(z_{1}, \ldots, z_{m}, y_{1}, \ldots, y_{m}, p\right) \in \mathbb{R}^{(l+m) S+l} \mid y_{i} \in L_{0}, i=1, \ldots, m\right\}
$$

for some $k$-dimensional subspace $\tilde{L}_{0}$ of $\mathbb{R}^{S}$. We claim that $\left(z_{1}^{0}, \ldots, z_{m}^{0}, p^{0}, \tilde{L}_{0}\right)$ is a pseudoequilibrium. Indeed, condition (iii) of Definition 4 follows directly from (6), and from (7) we get that $p^{0} \square z_{i}^{0} \in \tilde{L}_{0}$ for each $i$. Reasoning as before, and using that $z_{i}^{0} \in \tilde{L}_{0}$ for $i=1, \ldots, m$, we get that $p^{0} \cdot z_{i}^{0}=0$ for $i=1, \ldots, m$, so that $\phi\left(x^{0}\right) \cap \tilde{L}^{0}=\emptyset$ implies that $\sum_{i=1}^{m} z_{i}^{0}=0$. Also, from $\phi_{i}\left(x^{0}\right) \cap L_{0}$ for $i=1, \ldots, m$ we get that $P_{i}\left(z_{i}^{0}\right) \cap \tilde{L}_{0}=\emptyset, i=1, \ldots, m$, so that $\left(z^{0}, p^{0}, L^{0}\right)$ satisfies the conditions (i)-(iii) of Definition 4 and therefore is a pseudoequilibrium. 


\section{Concluding comments}

In the preceding sections, we have been concerned with equilibria which involve the choice of suitable subspaces, and it has been shown that the use of Plûcker coordinates makes it possible to show existence of equilibria by methods which are otherwise quite standard. This may be useful in the context of equilibrium theory, as shown by the two examples above, where we considered hierarchical equilibria, a generalization of the classical considerations of "minimum-wealth" conditions, as well as equilibria in economies with real assets, where the set if achievable net trades is determined by the current and future market prices. Here the subspaces must be considered as variables determined by the equilibrium conditions, and the parametrization offered makes this possible.

The results obtained can be extended in several directions. An extension which almost suggests itself is to allow for several subspaces in the formulation of an equilibrium. Here we should be interested in particular families of subspaces: A flag in $\mathbb{R}^{n}$ is a finite sequence $\left(L_{1}, L_{2}, \ldots, L_{k}\right)$ of subspaces of $\mathbb{R}^{n}$ with $L_{1} \subset \cdots \subset L_{k}$. Extending the previous results to equilibrium problems involving the choice of a suitable flag in in $\mathbb{R}^{n}$ might be worthwhile with a view to applications, actually it almost suggests itself in the context of hierarchical equilibria. However, such an extension is not as straightforward as those considered in the text, as a more elaborate method of parametrizing subspaces is needed. We shall not pursue this topic further at present.

\section{Appendix: Proof of Lemma 1}

The present section contains a proof of Lemma 1. The one given below is adapted from Merslyakov [21]. We need two additional lemmas.

Lemma 8 The map $W$ is continous, and if $z \in W\left(\left(\mathbb{R}^{d}\right)^{k}\right)$, then

$$
\sum_{t=1}^{k+1}(-1)^{t} z_{i_{1}, \ldots, i_{k-1}, j_{t}} z_{j_{1}, \ldots, j_{t-1}, j_{t+1}, \ldots, j_{k+1}}=0
$$

for all sets of indices $\left\{i_{1}, \ldots, i_{k-1}, j_{1}, \ldots, j_{k+1}\right\}$.

Proof: Continuity of $W$ follows directly from its definition (1). To prove the second statement, for each $t$ we expand the determinant as

$$
z_{i_{1}, \ldots, i_{k-1}, j_{t}}=\sum_{s=1}^{k}(-1)^{k+s} v_{s j_{t}} D_{i_{1}, \ldots, i_{k-1}, j_{t}}^{s, j_{t}},
$$

where $D_{i_{1}, \ldots, i_{k-1}, j_{t}}^{s, j_{t}}$ is the subdeterminant obtained by deleting column $j_{t}$ and row $s$. Then

$$
\begin{aligned}
\sum_{t=1}^{k+1} & (-1)_{i_{1}, \ldots, i_{k-1}, j_{t}}^{z} z_{j_{1}, \ldots, j_{t-1}, j_{t+1}, \ldots, j_{k+1}} \\
& =\sum_{t=1}^{k+1}(-1)^{t} \sum_{s=1}^{k}(-1)^{k+s} v_{s j_{t}} D_{i_{1}, \ldots, i_{k}-1, j_{t}}^{s, j_{t}} z_{j_{1}, \ldots, j_{t-1} j_{t+1}, \ldots, j_{k+1}} \\
& =\sum_{s=1}^{k}(-1)^{k+s-1} D_{i_{1}, \ldots, i_{k}-1, j_{t}}^{s, j_{t}} \sum_{t=1}^{k+1}(-1)^{t+1} v_{s j_{t}} z_{j_{1}, \ldots, j_{t-1}, j_{t+1}, \ldots, j_{k+1}} .
\end{aligned}
$$

Here the last sum can be identified as an expansion along the first row of the determinant

$$
\left|\begin{array}{ccc}
v_{s j_{1}} & \ldots & v_{s j_{k+1}} \\
v_{1 j_{1}} & \ldots & v_{1 j_{k+1}} \\
\vdots & & \vdots \\
v_{k j_{1}} & \ldots & v_{s j_{k+1}}
\end{array}\right|,
$$

and this determinant is zero as the matrix contains two identical rows. 
Lemma 9 If $z \in W\left(\left(\mathbb{R}^{d}\right)^{k}\right)$, then

$$
z_{i_{1}, \ldots, i_{k}} z_{j_{1}, \ldots, j_{k}}=\sum_{t=1}^{k} z_{i_{1}, \ldots, i_{s-1}, j_{t}, i_{s+1}, \ldots, i_{m}} z_{j_{1}, \ldots, j_{t-1}, i_{s}, j_{t+1}, \ldots, j_{k}}
$$

where $s$ is a fixed number with $1 \leq s \leq k$.

Proof: Apply Lemma 8 replacing $i_{1}, \ldots, i_{s-1}, i_{s}, \ldots, i_{m-1}, j_{1}, \ldots, j_{m}, j_{m+1}$ by $i_{1}, \ldots, i_{s-1}, i_{s+1}, \ldots, i_{m}$, $j_{1}, \ldots, j_{m}, i_{s}$ and use the skewsymmetry of $z_{i_{1}, \ldots, i_{k}}$ in the indices.

Proof of Lemma 1: Linearity follows immediately from the definition. For property (i), we notice that the matrix

$$
\left(\begin{array}{ccc}
u_{1 i_{1}} & \ldots & u_{1 i_{k}} \\
\vdots & & \vdots \\
u_{k i_{1}} & \ldots & u_{k i_{k}}
\end{array}\right)
$$

with $u_{j i_{h}}$ defined in (2), is a unit matrix when $z_{i_{1}, \ldots, i_{k}}=1$.

It remains only to show (ii), that is for each subset $\left\{j_{1}, \ldots, j_{k}\right\}$ of $\{1, \ldots, d\}$, we have

$$
\left|\begin{array}{ccc}
u_{1 j_{1}} & \ldots & u_{1 j_{k}} \\
\vdots & & \vdots \\
u_{k j_{1}} & \ldots & u_{k j_{k}}
\end{array}\right|=z_{j_{1}, \ldots, j_{k}} .
$$

We use induction on the number $\tau$ of indices in $\left\{j_{1}, \ldots, j_{k}\right\}$ which are not in the set $\left\{i_{1}, \ldots, i_{k}\right\}$. For $\tau=0$, the result follows from (i). Assume that 9 holds for some $0 \leq \tau<k$, and consider any subset $\left\{j_{1}, \ldots, j_{k}\right\}$ with $\tau+1$ indices not in $\left\{i_{1}, \ldots, i_{k}\right\}$.

Expanding the determinant in (9) after column $j_{s}$ we obtain

$$
\mathcal{D}=\sum_{t=1}^{k}(-1)^{s+t} u_{t j_{s}} D_{j_{1}, \ldots, j_{s-1}, j_{s+1}, \ldots, j_{k}}^{t, j_{s}} .
$$

Now,

$$
D_{j_{1}, \ldots, j_{s-1}, j_{s+1}, \ldots, j_{k}}^{s, j_{s}}=z_{j_{1}, \ldots, j_{s-1}, i_{s}, j_{s+1}, \ldots, j_{k}}
$$

by the induction hypothesis. For $t \neq s$,

$$
D_{j_{1}, \ldots, j_{s-1}, j_{s+1}, \ldots, j_{k}}^{t, j_{s}}=(-1)^{s+t} z_{j_{1}, \ldots, j_{s-1}, i_{t}, j_{s+1}, \ldots, j_{k}}
$$

(replace column $j_{s}$ by column $i_{t}$ and apply the induction hypothesis). Inserting in the expansion and using the definition of $u_{t j_{s}}$, one gets

$$
\begin{aligned}
\mathcal{D} & =\sum_{t=1}^{k} z_{i_{1}, \ldots, i_{t-1}, j_{s}, i_{t+1}, \ldots, i_{k}} z_{j_{1}, \ldots, j_{s-1}, i_{t}, j_{s+1}, \ldots, j_{k}} \\
& =z_{i_{1}, \ldots, i_{k}} z_{j_{1}, \ldots, j_{k}},
\end{aligned}
$$

where the last equality follows from Lemma 9. Since $z_{i_{1}, \ldots, i_{k}}=1$, the desired result is obtained.

\section{References}

1. E. Blum and W. Oettli, "From optimization and variational inequalities to equilibrium problems," Mathematical Student, vol. 63, pp. 123-145, 1994.

2. M. Bianchi and S. Schaible, "Generalized monotone bifunctions and equilibrium problems," Journal of Optimization Theory and Applications, vol. 90, pp. 31-43. 1996. 
3. A. N. Iusem and W. Sosa, "New existence results for equilibrium problems," Nonlinear Analysis, vol. 52, pp. 621-635, 2003.

4. Q. H. Ansari and F. Flores-Bazán, "Generalized vector quasi-equilibrium problems with applications," J. Math. Anal. Appl., vol. 277, pp. 246-256, 2003.

5. Z. Lin and J. Yu, "The existence of solutions for the system of generalized vector quasi-equilibrium problems," Applied Mathematics Letters, vol. 18, pp. 415-422, 2005.

6. P. Griffiths and J. Harris, Principles of Algebraic Geometry, Wiley, 1978.

7. E. Michael, "Continuous selections I," Annals of Mathematics, vol. 63, pp. 361-381, 1956.

8. D. Gale and A. Mas-Colell, "An equilibrium existence theorem for a general model without ordered preferences," Journal of Mathematical Economics, vol. 2, pp. 9-17, 1975.

9. S. Kakutani, "A generalization of Brouwer's fixed point theorem," Duke Mathematical Journal, vol. 8, pp. 457-459, 1941.

10. G. Debreu, Theory of value, Wiley, 1959.

11. V. I. Danilov and A. I. Sotskov, "A generalized economic equilibrium," Journal of Mathematical Economics, vol. 19, pp. 341-356, 1990.

12. M. Florig, "Arbitrary small indivisibilities," Economic Theory, vol. 22, pp. 831-843, 2002.

13. A. Konovalov and V. Marakulin, "Generalized equilibria in an economy without the survival assumption," EI Report 2002Đ49, Erasmus University Rotterdam, 2002.

14. D. Duffie and W. Shafer, "Equilibrium in incomplete markets: I. A basic model of generic existence," Journal of Mathematical Economics, vol. 14, pp. 285-300, 1985.

15. J. Geanakoplos, "An introduction to general equilibrium with incomplete asset markets," Journal of Mathematical Economics, vol. 19, pp. 1-38, 1990.

16. M. Magill and W. Shafer, "Incomplete markets," in: W. Hildenbrand and H. Sonnenschein (eds.), Handbook of Mathematical Economics, North Holland, 1991.

17. Y. Zhou, "Genericity Analysis on the Pseudo-Equilibrium Manifold," Journal of Economic Theory, vol. 73, pp. 79-92, 1997.

18. T. Momi, "Failure of the index theorem in an incomplete market economy," Journal of Mathematical Economics, vol. 48 , pp. 437-444, 2012.

19. M. Hoelle, M. Pireddu and A. Villanacci, "Incomplete financial markets with real assets and wealth-dependent credit limits," Journal of Economics, vol. 117, pp. 1-36, 2016.

20. O. Hart, "On the optimality of equilibrium when the market structure is incomplete," Journal of Economic Theory, vol. 11, pp. 418-443, 1975.

21. Yu. I. Merslyakov, Rational Groups (in Russian), Nauka, 1987. 